\title{
FORMAL FIBERS AND COMPLETE HOMOMORPHIC IMAGES
}

\author{
WILLIAM HEINZER AND CHRISTEL ROTTHAUS
}

(Communicated by Eric Friedlander)

\begin{abstract}
Let $(R, \mathbf{m})$ be an excellent normal local Henselian domain, and suppose that $\mathbf{q}$ is a prime ideal in $R$ of height $>1$. We show that, if $R / \mathbf{q}$ is not complete, then there are infinitely :nany height one prime ideals $\mathbf{p} \subseteq \mathbf{q} \widehat{R}$ of $\widehat{R}$ with $\mathrm{p} \cap R=0$; in particular, the dimension of the generic formal fiber of $R$ is at least one. This result may in fact indicate that a much stronger relationship between maximal ideals in the formal fibers of an excellent Henselian local ring and its complete homomorphic images is possibly satisfied. The second half of the paper is concerned with a property of excellent normal local Henselian domains $R$ with zero-dimensional formal fibers. We show that for such an $R$ one has the following good property with respect to intersection: for any field $L$ such that $\mathscr{Q}(R) \subseteq L \subseteq \mathscr{Q}(\widehat{R})$, the ring $L \cap \widehat{R}$ is a local Noetherian domain which has completion $\widehat{R}$.
\end{abstract}

\section{INTRODUCTION}

In [HRS] we established a correspondence between maximal ideals (resp. maximal ideals of maximal height) in the generic formal fiber of some Noetherian domains and between certain normal birational extensions of $R$. As shown there, it is possible to set up such a one-to-one correspondence if the local Noetherian domain $R$ has the following property:

The Complete homomorphic images property (CHI). Suppose $\mathbf{p} \subseteq \widehat{R}$ is a maximal ideal (resp. a maximal ideal of maximal height) in the generic formal fiber of $R$. We say that $R$ satisfies $(\mathrm{CHI})$ with respect for $\mathbf{p}$ if, for all but at most finitely many of the prime ideals $\mathbf{q} \supseteq \mathbf{p}$ in $\widehat{R}$, the ring $R /(\mathbf{q} \cap R)$ is complete. If this holds for all prime ideals $\mathbf{p}$ of $\widehat{R}$ that are maximal in the generic formal fiber of $R$, then we say that $R$ satisfies $(\mathrm{CHI})$.

In [HRS] we show that condition $(\mathrm{CHI})$ is satisfied for a number of excellent local domains. In fact, we know of no example of an excellent Henselian local

Received by the editors May 18, 1992.

1991 Mathematics Subject Classification. Primary 13B02, 13E05, 13F25, 13G05, 13H99, 13J10, $13 \mathrm{~J} 15$.

Key words and phrases. Generic formal fiber, approximation property, excellent local Henselian domain, complete homomorphic image, Noetherian intermediate ring.

The authors gratefully acknowledge partial support from the National Science Foundation while this work was done. The second author also thanks Purdue University for its hospitality during her visit. 
domain for which condition (CHI) fails. On the other hand, as we note in Example 1.8, condition ( $\mathrm{CHI}$ ) is not satisfied for the example given by Nagata [N, (E3.1) and (E5.1), pp. 206-208] of a local Noetherian domain with nonNoetherian integral closure. We also show in Examples 1.9 and 1.10 that there are excellent local domains $R$ for which condition $(\mathrm{CHI})$ is not satisfied, but the Henselization $R^{h}$ of $R$ in 1.9 and 1.10 does satisfy $(\mathrm{CHI})$.

The first section of this paper is concerned with a property of local Henselian domains $R$ which indicates that condition $(\mathrm{CHI})$ may be satisfied for the class of all excellent Henselian local rings. Using Artin approximation, we show in Theorem 1.2 that any height two prime ideal $\mathbf{q} \subseteq \widehat{R}$, for which the ring $R /(\mathbf{q} \cap R)$ is not complete, contains infinitely many height one prime ideals $\mathbf{p}_{n}$, $n \in \mathbb{N}$, which contract to $R$ in a minimal prime ideal. Following Matsumura in [M2], we use $\alpha(R)$ to denote the maximal dimension of the formal fibers of a local Noetherian ring $R$. It follows from Theorem 1.2 that if $R$ is a local Noetherian ring with the approximation property and with $\alpha(R)=0$ (i.e., the formal fibers of $R$ are zero-dimensional), then the ring $R / \mathbf{a}$ is complete for any ideal a in $R$ of height $>1$. Apparently a better measure of "closeness" of an excellent local domain $R$ to its completion $\widehat{R}$, but still $R^{h}<\widehat{R}$, is that its Henselization $R^{h}$ has complete homomorphic images modulo ideals of small height, rather than $R$, resp. $R^{h}$, be complete with respect to an ideal of large height.

We would like to point out that we do not know if Theorem 1.2 can be extended to prime ideals of larger height. By this we mean: is it true, for any excellent local Henselian domain $R$ and any prime ideal $\mathbf{q} \subseteq \widehat{R}$ with $\operatorname{ht}(\mathbf{q})=t>1$ and the ring $R /(\mathbf{q} \cap R)$ not complete, that the ideal $\mathbf{q}$ contains infinitely many prime ideals of height $t-1$ which are in the generic formal fiber of $R$ ? It seems to us that this question and the question of whether every local excellent Henselian ring has (CHI) may very well be related, and an affirmative answer to one of them may lead to an affirmative answer to the other.

In [R2] an excellent regular local ring $R$ has been constructed which has the following interesting property:

The Noetherian intermediate rings property (NIR). For any field $L$ such that $\mathscr{Q}(R) \subseteq L \subseteq \mathscr{Q}(\widehat{R})$, the local ring $\widehat{R} \cap L$ is Noetherian and has completion $\widehat{R}$.

In the second part of this paper we show that condition (NIR) is satisfied by every excellent normal local Henselian domain with zero-dimensional formal fibers. Although we do not know if there is a descent from the Henselization to obtain (NIR) in the case where the ring is not Henselian, we show in Theorem 2.11 that condition (NIR) is attained by analytically normal local Noetherian domains which have $\alpha(R)=0$ and satisfy condition $(\mathrm{CHI})$.

We use $\mathscr{Q}(R)$ to denote the field of fractions of an integral domain $R$.

\section{COMPLETE HOMOMORPHIC IMAGES}

We are interested in studying certain properties of rings with the approximation property, i.e., local Noetherian rings which satisfy the conditions of the following definition:

Definition 1.1. Let $(R, \mathbf{m})$ be a local Noetherian ring, and let $\widehat{R}$ be the completion of $R$ with respect to its maximal ideal $\mathbf{m}$. $R$ has the approximation 
property if every system of equations defined over $R$, which is solvable in $\widehat{R}$, is already solvable in $R$. More precisely, the following holds true:

Let $X=\left(X_{1}, \ldots, X_{n}\right)$ be variables, and let $(f)=\left(f_{1}, \ldots, f_{m}\right) \subseteq R[X]$ be a system of polynomials over $R$. Suppose the system of equations $f=0$ has a solution $\hat{y}=\left(\hat{y}_{1}, \ldots, \hat{y}_{n}\right) \in \widehat{R}^{n}$. Then there is an element $y=\left(y_{1}, \ldots, y_{n}\right) \in$ $R^{n}$ with $f(y)=0$.

It is well known [R2] that local rings with the approximation property are excellent and Henselian. On the other hand it was shown in [R1] that excellent local Henselian rings, which contain the rational numbers, have the approximation property. The approximation property for excellent Henselian rings has also been studied by Popescu [P1, P2]. Apparently, André [An] has recently shown, by using some of Popescu's ideas, that excellent local Henselian rings which contain a field have the approximation property.

Theorem 1.2. Let $R$ be a local Noetherian domain with the approximation property, and let $\mathbf{q} \subseteq R$ be a prime ideal in $R$ of height two. Suppose that the ring $R / \mathbf{q}$ is not complete. Then there are infinitely many mutually distinct prime ideals $\mathbf{p}_{n} \subseteq \widehat{R}, n \in \mathbb{N}$, with the following properties:

(i) $\mathbf{p}_{n} \subseteq \mathbf{q} \widehat{R}$.

(ii) $\mathbf{p}_{n} \cap R=(0)$.

Proof. We first note that since $R$ is Henselian and excellent, the ideal $\mathbf{q} \widehat{R}$ is a prime ideal in $\widehat{R}$. We pick elements $x, y \in \mathbf{q}$ such that $\mathrm{ht}(x, y)=2$. Let $w \in \widehat{R}$ be an invertible element such that the residue class $w^{\prime}$ of $w$ in $\widehat{R} / \mathbf{q} \widehat{R}$ is not in $R / \mathbf{q}$.

Let $\mathbf{p}_{n} \subseteq \mathbf{q} \widehat{R}$ be a prime ideal of height one in $\widehat{R}$, which contains the element $x^{n} w+y$. Since the ideal $(x, y) \widehat{R}$ has height two and since $w$ is invertible in $\widehat{R}$, the prime ideals $\mathbf{p}_{n}$ are mutually distinct. We want to show that the prime ideals $\mathbf{p}_{n}$ are in the generic formal fiber of the ring $R$. We fix some $m \in \mathbb{N}$ and identify the prime ideal $\mathbf{p}_{m}$ with $\mathbf{p}$ in the following.

Assume that $\mathbf{p} \cap R \neq(0)$. Then $\mathbf{p}=(\mathbf{p} \cap R) \widehat{R}$. Let $p_{1}, \ldots, p_{r} \in R$ be generators of $\mathbf{p}$. We can find elements $\hat{a}_{i} \in \widehat{R}$ such that

$$
x^{m} w+y=\sum_{i=1}^{r} \hat{a}_{i} p_{i} .
$$

Since $R$ has the approximation property, for every integer $n \in \mathbb{N}$, we can find elements $w_{n}$ and $a_{i n} \in R$ such that

$$
x^{m} w_{n}+y=\sum_{i=1}^{r} a_{i n} p_{i}, \quad w-w_{n} \in \hat{\mathbf{m}}^{n}, \quad \text { and } \quad \hat{a}_{i}-a_{i n} \in \hat{\mathbf{m}}^{n} .
$$

This implies

$$
x^{m}\left(w-w_{n}\right) \in \mathbf{p} \widehat{R} \quad \text { for all } n \in \mathbb{N} .
$$

Since $\mathbf{p}$ is a height one prime ideal and since, by assumption, $h t(x, y)=2$, we conclude

$$
w-w_{n} \in \mathbf{p} \text { for all } n \in \mathbb{N} .
$$

But this implies also $w-w_{n} \in \mathbf{q} \widehat{R}$ for all $n \in \mathbb{N}$, and hence $w^{\prime}$ equals the residue class of $w_{n}$ in $R / \mathbf{q}$. This contradiction completes the proof. 
Corollary 1.3. Let $R$ be a local domain with the approximation property, and let $\mathbf{q} \subseteq \widehat{R}$ be a prime ideal of height $>1$. Suppose that the ring $R /(\mathbf{q} \cap R)$ is not complete. Then there are infinitely many height one prime ideals $\mathbf{p} \subset \mathbf{q}$ with $\mathbf{p} \cap R=0$.

Proof. We can assume $\operatorname{ht}(\mathbf{q})=2$. If $\mathbf{q}$ is extended from $R$, Theorem 1.2 applies. If $\mathbf{q}$ is not extended from $R$, the intersection $\mathbf{q} \cap R$ must be a prime ideal of height $<2$, and all but at most finitely many of the height one prime ideals of $\widehat{R}$ contained in $\mathbf{q}$ must contract in $R$ to $(0)$.

Similarly, we see immediately

Corollary 1.4. Let $R$ be a local ring with the approximation property, and let $\mathbf{q}$ be a prime ideal in $\widehat{R}$ with $\mathrm{ht}(\mathbf{q})>1$. Suppose that the ring $R /(\mathbf{q} \cap R)$ is not complete. Then for every minimal prime ideal $\mathbf{w} \subset \mathbf{q}$ there are infinitely many height one prime ideals $\mathbf{p}$ with $\mathbf{w} \subset \mathbf{p} \subset \mathbf{q}$ such that $\mathbf{p} \cap R$ is the minimal prime $\mathbf{w} \cap R$ of $R$.

The possible dimensions of the formal fibers of a local Noetherian ring are considered in [M2] and [R3]. In [R3] an excellent regular local ring $R_{s}$ has been constructed which has the following properties:

(a) The completion $\widehat{R}_{s}$ of $R_{s}$ has infinite transcendence degree over $R_{s}$.

(b) For every nonzero ideal $\mathbf{a} \subseteq R_{s}$, the ring $R_{s} / \mathbf{a}$ is complete, and $\alpha\left(R_{s}\right)$ $=0$.

We would like to note that if $R_{s}$ is the regular local ring from [R3], then the ring $R_{s}[[w]]$, where $w$ is a variable, is another example of an excellent regular local ring with zero-dimensional formal fiber. But the height one prime $\mathbf{p}=w R_{s}[[w]]$ gives a factor ring $R_{s}[[w]] / \mathbf{p} \cong R_{s}$ that is not complete. Rather than condition (b), the ring $R_{s}[[w]]$, and its Henselization, satisfy the slightly weaker condition

$\left(\mathbf{b}^{\prime}\right)$ For all but finitely many prime ideals $\mathbf{p} \subseteq R_{s}[[w]]$, the ring $R_{s}[[w]] / \mathbf{p}$ is complete.

It would be interesting to know whether every excellent Henselian local domain $R$ with $\alpha(R)=0$ satisfies condition $\left(\mathrm{b}^{\prime}\right)$. So far we have only obtained from Theorem 1.2

Corollary 1.5. Let $R$ be a local ring with the approximation property and with $\alpha(R)=0$, and let $\mathbf{a} \subseteq \widehat{R}$ be an ideal of height $>1$. Then

(i) the ideal a is extended from $R$,

(ii) the ring $R /(\mathbf{a} \cap R)$ is complete.

Proof. Every prime ideal $\mathbf{q} \subseteq \widehat{R}$ containing a has $\mathrm{ht}(\mathbf{q})>1$. Since $\alpha(R)=$ 0 , Corollary 1.4 implies that the ring $R /(\mathbf{q} \cap R)$ is a complete local domain. Therefore, $R /(\mathbf{a} \cap R)$ has the property that modulo any minimal prime it is a complete local domain. It follows (cf. [HRS, Corollary 1.10]) that $R /(\mathbf{a} \cap R)$ is complete. Thus, $(\mathbf{a} \cap R) \hat{R}=\mathbf{a}$, and $\mathbf{a}$ is also extended from $R$.

Corollary 1.6. Let $R$ be a local domain with the approximation property and with $\alpha(R)=0$. Suppose that $R$ is complete with respect to the a-adic topology for some nonzero ideal a $\subset R$. Then $R$ satisfies condition $(\mathrm{CHI})$.

Proof. Let $\mathbf{q} \subseteq \widehat{R}$ be a prime ideal of height one that does not contain $\mathbf{a}$. Then the ideal $\left(\mathbf{q}+\mathbf{a}^{n}\right) \widehat{R}$ is of height at least two for every $n \in \mathbb{N}$. By Corollary 1.5 
the ring $R /\left(\left(\mathbf{q}+\mathbf{a}^{n}\right) \widehat{R} \cap R\right)$ is complete, but

$$
R /\left(\left(\mathbf{q}+\mathbf{a}^{n}\right) \widehat{R} \cap R\right)=R /\left((\mathbf{q} \cap R)+\mathbf{a}^{n}\right) .
$$

Since $\lim \left(R /\left((\mathbf{q} \cap R)+\mathbf{a}^{n}\right)\right)$ is the a-adic completion of $R /(\mathbf{q} \cap R)$ and since $R$ is a-adically complete, we see that the ring $R /(\mathbf{q} \cap R)$ is complete. Therefore, $R$ satisfies condition $(\mathrm{CHI})$.

Example 1.7. Let $R_{s}$ be the excellent regular local ring of [R3] with $\alpha\left(R_{S}\right)=0$. The power series extension $R_{s}[[w]]$ of $R_{s}$ is another example of a regular local ring with $\alpha\left(R_{s}[[w]]\right)=0$. Although $R_{s}$ and $R_{s}[[w]]$ are possibly not Henselian, they both satisfy condition (CHI). We would like to note that by adjoining more variables the dimension of the formal fiber increases. For example, the ring $R_{s}[[w, v]]$ has a generic formal fiber of dimension one.

Example 1.8. Nagata in [N, (E3.1) and (E5.1), pp. 206-208] has the following construction. Let $k$ be a field of characteristic $p>0$ such that $\left[k: k^{p}\right]=\infty$; let $x_{1}, \ldots, x_{n}$ be indeterminates; and let $R_{n}=k^{p}\left[\left[x_{1}, \ldots, x_{n}\right]\right][k]$. Nagata shows that $R_{n}$ is an $n$-dimensional regular local ring with completion $\widehat{R}_{n}=k\left[\left[x_{1}, \ldots, x_{n}\right]\right]$ and that $R_{n}<\widehat{R}_{n}$ with $\widehat{R}_{n}$ a purely inseparable integral extension of $R_{n}$. It follows that $R_{n}$ is Henselian and that the formal fibers of $R_{n}$ are zero-dimensional. Moreover, $R_{n}$ is not a Nagata ring (cf. [M3, Theorem 71, p. 237]). It is clear from the construction that, for $t$ with $0<t<n$, we have $R_{t} \cong R_{n} /\left(x_{t+1}, \ldots, x_{n}\right) R_{n}$. This shows that Theorem 1.2 is not true for an arbitrary local Noetherian Henselian domain.

Example 1.9. Let $k$ be a field of characteristic zero, and let $x$ be a variable over $k$. We consider the extension of discrete valuation rings $k[x]_{(x)} \subseteq k[[x]]$ and take a transcendence basis $\left(w_{i}\right)_{i \in I} \subseteq k[[x]]$ of $k((x))$ over $k(x)$. We define $D=k\left(x, w_{i}\right)_{i \in I} \cap k[[x]]$. Obviously, $D$ is a discrete valuation ring with completion $k[[x]]$. Since $D$ contains a field of characteristic zero, $D$ is excellent. Moreover, it follows from a theorem by Schmidt [BKKN, (2.3.11)] that $D$ is not Henselian, and hence $D$ is not complete. Let $R$ be the ring $D[y]_{(x, y)}$, where $y$ is another variable. Obviously, $R$ is an excellent local domain with completion $k[[x, y]]$. It is equally easy to see that $R$ is contained in the ring $k[[x]][y]_{(x, y)}$, which is algebraic over $R$. This implies, in particular, that $\alpha(R)=0$. For rings $R$ with $\alpha(R)=0$, condition (CHI) requires that the ring $R / \mathbf{p}$ be complete for all but finitely many prime ideals $\mathbf{p} \subseteq R$. But this is not satisfied for the ring $R$ constructed above; namely, take any element $d \in(x) D$. Then the prime ideal $(y-d) R \subseteq R$ is such that $R /(y-d) R=D$. Thus $R$ is an example of an excellent local ring which does not satisfy condition $(\mathrm{CHI})$. On the other hand, its Henselization $R^{h}$ contains the ring $k[[x]][y]_{(x, y)}$ and hence satisfies $(\mathrm{CHI})$.

Example 1.10. In order to construct an excellent local domain $R$ with $\alpha(R)>0$ which does not satisfy condition $(\mathrm{CHI})$, we modify the example from 1.9 by adjoining more variables. So let $R_{m}=D\left[y_{1}, \ldots, y_{m}\right]_{\left(x, y_{1}, \ldots, y_{m}\right)}$. Moreover, we assume that the field $k$ is the field of rational numbers $\mathbb{Q}$. Additionally, we pick the transcendence basis $\left(w_{i}\right)_{i \in I} \subseteq k[[x]]$ such that it contains the algebraically independent set $e^{x^{n}}, n \in \mathbb{N}$. A similar argument as in Example 1.9 shows that $\alpha\left(R_{m}\right)=\operatorname{dim}\left(R_{m}\right)-2=m-1$. As shown in [M2, Example 2, p. 
264], the prime ideal $\mathbf{p}=\left(y_{2}-y_{1} e^{y_{1}^{2}}, \ldots, y_{m}-y_{1} e^{y_{1}^{m}}\right) \subseteq k\left[\left[x, y_{1}, \ldots, y_{m}\right]\right]$ is maximal in the generic formal fiber of the ring $k[[x]]\left[y_{1}, \ldots, y_{m}\right]_{\left(x, y_{1}, \ldots, y_{m}\right)}$. Since the Henselization of $R_{m}$ contains the ring $k[[x]]\left[y_{1}, \ldots, y_{m}\right]_{\left(x, y_{1}, \ldots, y_{m}\right)}$, the prime ideal $\mathbf{p}$ is also maximal in the generic formal fiber of

$$
R_{m}=D\left[y_{1}, \ldots, y_{m}\right]_{\left(x, y_{1}, \ldots, y_{m}\right)} .
$$

We consider the prime ideals $\mathbf{q}_{n}=\left(y_{1}-x^{n}, \mathbf{p}\right)$ in $k\left[\left[x, y_{1}, \ldots, y_{m}\right]\right]$. Since $\mathbf{q}_{n}=\left(y_{1}-x^{n}, y_{2}-x^{n} e^{x^{2 n}}, \ldots, y_{m}-x^{n} e^{x^{m n}}\right)$, obviously $\mathbf{q}_{n}$ is extended from $R_{m}$ and $R_{m} /\left(\mathbf{q}_{n} \cap R_{m}\right)=D$. Thus there are infinitely many prime ideals $\mathbf{q}_{n} \supseteq \mathbf{p}$ of $\widehat{R}_{m}$ such that $R_{m} /\left(\mathbf{q}_{n} \cap R_{m}\right)$ is not complete, so $R_{m}$ does not satisfy condition (CHI). Again, however, the Henselization of $R_{m}$ does satisfy condition (CHI).

These examples demonstrate that condition ( $\mathrm{CHI}$ ) could only possibly be true in general for excellent Henselian local rings. Since we do not know if Artin approximation can provide a more general result than Theorem 1.2, in the remainder of this section we present several results which do not involve approximation.

Proposition 1.11. Suppose $(R, \mathbf{m})$ is a local Noetherian ring that is complete with respect to an ideal a of positive height. If $\mathbf{p} \subseteq \widehat{R}$ is a prime ideal such that $\operatorname{dim}(\widehat{R} / \mathbf{p})=2$ and $\mathbf{p} \cap R$ is a minimal prime of $R$, then $R$ satisfies $(\mathrm{CHI})$ with respect to $\mathbf{p}$.

Proof. Since $\mathbf{p} \cap R$ is a minimal prime of $R, \mathbf{a}^{n}$ is not contained in $\mathbf{p}$ for any $n \in \mathbb{N}$. If $\left(\mathbf{p}+\mathbf{a}^{n}\right) \widehat{R}$ is $\mathbf{m} \widehat{R}$-primary, then $\mathbf{p}$ is extended from $R$ by [HRS, Lemma 1.15], and hence $R /(\mathbf{p} \cap R)$ is complete, so $R$ has $(\mathrm{CHI})$ with respect to $\mathbf{p}$. Suppose $\left(\mathbf{p}+\mathbf{a}^{n}\right) \widehat{R}$ is not $\mathbf{m} \widehat{R}$-primary. If $\mathbf{q}$ is a prime ideal with $\mathbf{p}<\mathbf{q}<\mathbf{m} \widehat{R}$ and $\mathbf{a}$ is not contained in $\mathbf{q}$, then $\left(\mathbf{q}+\mathbf{a}^{n}\right) \widehat{R}$ is $\mathbf{m} \widehat{R}$-primary, and by a similar argument to that above using [HRS, Lemma 1.15], we see that $q$ is extended from $R$ and $R /(\mathbf{q} \cap R)$ is complete.

Suppose $(R, \mathbf{m})$ is a local Noetherian domain with completion $\widehat{R}$ and $\mathbf{q}$ is a prime ideal of $\widehat{R}$ such that $R /(\mathbf{q} \cap R)$ is not complete. We are interested in what we can say about the existence of prime ideals of $\widehat{R}$ that are contained in $\mathbf{q}$ and are in the generic formal fiber of $R$. If we set $A=R_{\mathbf{q} \cap R}$ and $B=\widehat{R}_{\mathbf{q}}$, then $B$ is a local Noetherian ring that dominates and is faithfully flat over the local Noetherian domain $A$, and the prime ideals contained in $\mathbf{q}$ and in the generic formal fiber of $R$ correspond in a natural one-to-one way to the prime ideals of $B$ in the generic fiber over $A$.

If $\mathbf{q}$ is extended from $R$, so $(\mathbf{q} \cap R) \widehat{R}=\mathbf{q}$, then the completion of $R /(\mathbf{q} \cap R)$ is $\widehat{R} / \mathbf{q}$. If, moreover, $R$ is excellent and Henselian, and if $R /(\mathbf{q} \cap R)$ is not complete, then we have $R /(\mathbf{q} \cap R)<\widehat{R} / \mathbf{q}$, and since $R /(\mathbf{q} \cap R)$ is excellent and Henselian, it is algebraically closed in its completion. Therefore, Theorem 1.12, with $A=R_{\mathbf{q} \cap R}$ and $B=\widehat{R}_{\mathbf{q}}$, gives an alternative proof for Theorem 1.2 in the case where $\widehat{R}_{\mathbf{q}}$ is normal.

Theorem 1.12. Suppose $(A, \mathbf{m})$ is a two-dimensional normal local Noetherian domain that is analytically irreducible and $(B, \mathbf{n})$ is a normal local Noetherian domain that dominates $A$ and is faithfully flat over $A$. If $B$ is not algebraic 
over $A$ and also if $B / \mathbf{n}$ as a field extension of $A / \mathbf{m}$ is not algebraic, then there exist infinitely many height one prime ideals of $B$ in the generic fiber over $A$.

Proof. Choose $t \in B$ such that the image of $t$ in $B / \mathbf{n}$ is transcendental over $A / \mathbf{m}$. Modifying $t$ if necessary by an element of $\mathbf{n}$, we may assume that $t$ is transcendental over $A$. Since the residue of $t$ in $B / \mathbf{n}$ is transcendental over $A / \mathbf{m}$, we have $\mathbf{m} A[t]=A[t] \cap \mathbf{n}$. Therefore, $(B, \mathbf{n})$ dominates $A(t)=A[t]_{\mathbf{m}[t]}$, the "Kronecker" extension of $A$ obtained by localizing $A[t]$ at the multiplicative set of polynomials in $A[t]$ that have a unit coefficient. We note that $A(t)$ is a two-dimensional normal local domain with completion $\widehat{A(t)}$ which is the completion of $\widehat{A}(t)$, and $A$ being analytically irreducible implies that $A(t)$ is analytically irreducible. Let $C=B \cap \mathscr{Q}(A)(t)$. Then $C$ is a normal local Krull domain that birationally dominates $A(t)$. Moreover, every height one prime of $C$ is the contraction of a height one prime of $B$, and the fact that $B$ is faithfully flat over $A$ implies that the maximal ideal $\mathrm{m}$ of $A$ is not contained in any height one prime of $B$. Since $A(t)$ is a two-dimensional Noetherian domain, $C$ is Noetherian, and $\operatorname{dim}(C) \leq 2$ [Ni, Corollary 3, p. 398]. Therefore, $\mathrm{m} C$ is primary for the maximal ideal of $C$. Since $A(t)$ is analytically irreducible, it follows that $A(t)=C$ [N, (37.4), p. 137; C, Theorem 3, p. 663]. Since there are infinitely many height one primes of $A(t)$ in the generic fiber over $A$ and since each height one prime of $C=B \cap \mathscr{Q}(A)(t)$ is the contraction of a height one prime of $B$, we conclude that there are infinitely many height one primes in the generic fiber of $B$ over $A$.

Corollary 1.13. Suppose $(R, \mathbf{m})$ is an excellent normal local Henselian domain. If $\mathbf{q}$ is a prime ideal of $R$ of height $\geq 2$ such that $R / \mathbf{q}$ is not complete, then there exist infinitely many prime ideals of $\widehat{R}$ that are in the generic formal fiber of $R$ and are contained in $\mathbf{q} \widehat{R}$. Thus if $\alpha(R)=0$, then, for any ideal $\mathbf{a} \subseteq \widehat{R}$ with $\mathrm{ht}(\mathbf{a}) \geq 2$, the ring $R /(\mathbf{a} \cap R)$ is complete and a is extended from $R$.

Proof. If $R / \mathbf{q}$ is not complete, then, for any prime ideal $\mathbf{q}_{0} \subseteq \mathbf{q}$, we also have that $R / \mathbf{q}_{0}$ is not complete since $R / \mathbf{q}$ is a homomorphic image of $R / \mathbf{q}_{0}$. Therefore, we may assume that $\operatorname{ht}(\mathbf{q})=2$. With $A=R_{\mathbf{q}}$ and $B=\widehat{R}_{\mathbf{q} \widehat{R}}$, Theorem 1.12 implies the existence of infinitely many prime ideals in $\mathbf{q} \widehat{R}$ that are in the generic formal fiber of $R$. Thus $\alpha(R)=0$ implies that $R / \mathbf{q}$ is complete for each prime ideal $\mathbf{q}$ in $R$ of height $\geq 2$. It follows (cf. [HRS, Corollary 1.10]) that $R /(\mathbf{a} \cap R)$ is complete and $(\mathbf{a} \cap R) \widehat{R}=\mathbf{a}$ for each ideal $\mathbf{a}$ of $\widehat{R}$ with $h t(a) \geq 2$.

\section{INTERMEDIATE NOETHERIAN RINGS}

In this section we continue our investigation of excellent local Henselian rings $R$ with zero-dimensional formal fibers. We show that these rings, if they are integrally closed, have condition (NIR), i.e., for any field $L$ such that $\mathscr{Q}(R) \subseteq L \subseteq \mathscr{Q}(\widehat{R})$, the intermediate ring $D=L \cap \widehat{R}$ is local Noetherian with completion $\widehat{R}$.

We begin with a result in a more general setting.

Theorem 2.1. Suppose $A \subseteq B$ are integral domains, and assume that:

(i) every ideal in $A$ is contracted from $B$,

(ii) every principal ideal in $B$ is the extension of a principal ideal in $A$. 
If $L$ is a field such that $\mathscr{Q}(A) \subseteq L \subseteq \mathscr{Q}(B)$ and $D=B \cap L$, then every ideal in $D$ is contracted from $B$, and the map $\mathbf{d} \rightarrow \mathbf{d} B$ defines a one-to-one inclusion preserving correspondence between the ideals of $D$ and the ideals of $B$. In particular, if $B$ is Noetherian, then $D$ is Noetherian.

Proof. We note that the condition that every principal ideal of $B$ is the extension of a principal ideal of $A$ implies that every ideal in $B$ is extended from $A$. Since by hypothesis every ideal in $A$ is contracted from $B$, it follows that the ideals of $A$ and $B$ are in one-to-one correspondence with respect to extension and contraction.

If $d \in D$, then by hypothesis $d B=a B$ for some $a \in A$. Since $D=B \cap L$, we have

$$
d D=d B \cap D=a B \cap D=a D .
$$

It follows that every ideal of $D$ is extended from $A$. Let $I$ be an ideal in $D$. Then $I=(I \cap A) D$, so $I B=(I \cap A) B$. Since every ideal in $A$ is contracted from $B, I B \cap A=I \cap A$. Let $J=I B \cap D$. Then $I B=J B$, so

$$
I \cap A \subseteq J \cap A \subseteq J B \cap A=I B \cap A=I \cap A .
$$

Therefore, $I=(I \cap A) D=(J \cap A) D=J$. We conclude that every ideal of $D$ is contracted from $B$. Since every ideal in $B$ is extended from $A$, every ideal in $B$ is extended from $D$, and the other assertions of the theorem follow.

Remark 2.2. We remark that if $B$ is local, then any principal ideal of $B$ that is extended from $A$ is the extension of a principal ideal of $A$; and the condition that every ideal of $A$ is contracted from $B$ is satisfied if $B$ is faithfully flat over $A$.

Corollary 2.3. Let $(R, \mathbf{m})$ be an analytically irreducible local Noetherian domain with $\alpha(R)=0$. Suppose additionally that every ideal in $\widehat{R}$ is extended from $R$. Then $R$ satisfies condition (NIR), i.e., for any field $L$ such that $\mathscr{Q}(R) \subseteq L \subseteq$ $\mathscr{Q}(\widehat{R})$, the ring $D=\widehat{R} \cap L$ is local Noetherian with completion $\widehat{D}=\widehat{R}$.

Proof. The hypotheses imply that the conditions of Theorem 2.1 are satisfied. Since every ideal in $D$ is contracted from $\widehat{R}$, it follows that $\mathbf{m} D$ is the maximal ideal of $D$ and $\widehat{D}=\widehat{R}$.

Example 2.4. Corollary 2.3 applies to the example of the excellent regular local ring $R_{s}$ constructed in [R3]. But it does not apply to Nagata's example mentioned in Example 1.8. As Nagata has shown, if $R=k^{2}[[x, y, z]][k]$, where $k$ is a field of characteristic 2 with $\left[k: k^{2}\right]=\infty$, then for an appropriate element $d \in \widehat{R}-R$ the integral closure $T$ of the ring $R[d]$ is not Noetherian. Since $T=\mathscr{Q}(R)(d) \cap \widehat{R}$, the Noetherian intermediate rings property (NIR) does not hold for this $R$.

In order to apply Theorem 2.1 to a broader class of excellent local rings, we note the following facts about ideals in $\widehat{R}$ being extended from $R$ :

Proposition 2.5. Let $(R, \mathbf{m})$ be an excellent Henselian local domain with $\alpha(R)=$ 0 . Suppose that $\mathbf{q} \subseteq \widehat{R}$ is a prime ideal of height one such that the ring $\widehat{R}_{\mathbf{q}}$ is regular. Let $\mathbf{w} \subseteq \mathbf{q}$ be a $\mathbf{q}$-primary ideal of $\widehat{R}$. Then $\mathbf{w}$ is extended from $R$. 
Proof. Since $R$ is Henselian with $\alpha(R)=0$, the prime ideal $\mathbf{q}$ is extended from $R$. We put $\mathbf{q}_{0}=\mathbf{q} \cap R$ and $\mathbf{w}_{0}=\mathbf{w} \cap R$. Obviously, the ideal $\mathbf{w}_{0}$ is $\mathbf{q}_{0}$-primary. We consider the induced morphism of discrete valuation rings $R_{\mathbf{q}_{0}} \rightarrow \widehat{R}_{\mathbf{q}}$. Suppose the element $u \in \mathbf{q}_{0}$ is such that $\mathbf{q}_{0} R_{\mathbf{q}_{0}}=u R_{\mathbf{q}_{0}}$. Then we also have $\mathbf{q} \widehat{R}_{\mathbf{q}}=u \widehat{R}_{\mathbf{q}}$, and there is an integer $l \in \mathbb{N}$ such that $\mathbf{w} \widehat{R}_{\mathbf{q}}=u^{l} \widehat{R}_{\mathbf{q}}$. This implies $u^{l} \in \mathbf{w} \cap R=\mathbf{w}_{0}$ and, hence, $\mathbf{w}_{0} \widehat{R}_{\mathbf{q}}=\mathbf{w} \widehat{R}_{\mathbf{q}}$. Since $R$ is excellent Henselian, the ideal $\mathbf{w}_{0} \widehat{R}$ is q-primary, and thus $\mathbf{w}_{0} \widehat{R}=\mathbf{w}$.

Corollary 2.6. Let $R$ be an excellent normal local Henselian domain with $\alpha(R)$ $=0$. Then every ideal of $\widehat{R}$ is extended from $R$.

Proof. By Corollary 1.13 every ideal of $\widehat{R}$ of height $>1$ is extended from $R$, and by the previous proposition any unmixed ideal of $\widehat{R}$ of height one is extended. Thus every ideal of $\widehat{R}$ is extended from $R$.

Corollary 2.7. Let $(R, \mathbf{m})$ be an excellent normal local Henselian domain with $\alpha(R)=0$. Then $R$ satisfies condition (NIR), i.e., for any field $L$ such that $\mathscr{Q}(R) \subseteq L \subseteq \mathscr{Q}(\widehat{R})$, the ring $D=L \cap \widehat{R}$ is a normal local Noetherian domain with completion $\widehat{R}$. If, additionally, the rational numbers $\mathbb{Q}$ are contained in $R$, then $D$ is excellent.

Proof. By Corollary 2.6 the assumptions of Corollary 2.3 are satisfied. The formal fibers of $D$ are zero-dimensional, and every ideal of $\widehat{D}=\widehat{R}$ is extended from $D$. Thus, $D$ is excellent if $\mathbb{Q} \subseteq D$.

Question 2.8. We do not know an example of an excellent local domain $R$ which contains a field of positive characteristic and is such that $\alpha(R)=0$, $\operatorname{dim}(R) \geq 3$, and $R<\widehat{R}$.

Remark 2.9. It is very well possible that the Noetherian intermediate rings property is satisfied for a larger class of local Noetherian rings. For example, Valabrega has shown in [V, Proposition 3, p. 3] that, if $C$ is a DVR and $y$ an indeterminate, then for any field $L$ such that $\mathscr{Q}(C[y]) \subseteq L \subseteq \mathscr{Q}(C[[y]])$ the ring $D=L \cap C[[y]]$ is a regular local ring with completion $\hat{C}[[y]]$. Thus, if $k$ is a field and $x, y$ are variables, then for any field $L$ such that $k(x, y) \subseteq$ $L \subseteq \mathscr{Q}(k[x][[y]])$ the ring $D=L \cap k[[x, y]]$ is a two-dimensional regular local ring with completion $k[[x, y]]$. It would be interesting to know if Valabrega's result can be extended to local Noetherian domains $R$ (assume excellent and Henselian if necessary) with $\alpha(R)=1$ and intermediate fields

$$
\mathscr{Q}(R) \subseteq L \subseteq \mathscr{Q}\left((R, t)^{\wedge}\right),
$$

where $(R, t)^{\wedge}$ is the completion of $R$ with respect to the principal ideal $(t) \subseteq$ $R$. In general, for a field $L$ such that $k(x, y) \subseteq L \subseteq \mathscr{Q}(k[[x, y]])$, it can happen that $D=L \cap k[[x, y]]$ is three-dimensional and, therefore, does not have completion $k[[x, y]]$ (cf. [HR]).

In case $R$ is a local Noetherian ring which satisfies $\alpha(R)=0$ and condition (CHI), we obtain that for all but at most finitely many prime ideals $\mathbf{p} \subseteq \widehat{R}$ the ring $R /(\mathbf{p} \cap R)$ is complete. If we assume additionally that $R$ is analytically normal, we can conclude that $R$ also satisfies condition (NIR) by using the following slightly weaker version of a theorem by Nishimura [Ni] on Krull domains: 
Lemma 2.10 [HRS, (1.5)]. Let $D$ be a Krull domain. If $D / \mathbf{p}$ is Noetherian for all but at most finitely many of the height one prime ideals $\mathbf{p}$ of $D$, then $D$ is Noetherian.

Theorem 2.11. Suppose $(R, \mathbf{m})$ is an analytically normal local Noetherian domain with $\alpha(R)=0$. If $R$ satisfies (CHI), then $R$ also satisfies (NIR), i.e., for every field $L$ such that $\mathscr{Q}(R) \subseteq L \subseteq \mathscr{Q}(\widehat{R})$, the ring $D=L \cap \widehat{R}$ is normal local Noetherian with completion $\widehat{D}=\widehat{R}$.

Proof. Obviously, the ring $D$ is a Krull domain. We want to show that $D$ satisfies the assumptions of Lemma 2.10. Let $\mathbf{p}_{1}, \ldots, \mathbf{p}_{n}$ be the prime ideals in $\widehat{R}$ for which the ring $R /\left(\mathbf{p}_{i} \cap R\right)$ is not complete, and let $\mathbf{q}_{1}, \ldots, \mathbf{q}_{s}$ be their contractions to $D$. We take a height one prime ideal $\mathbf{q} \subseteq D$ which is different from the $\mathbf{q}_{i}$. Since every height one prime ideal of $D$ is the contraction of a height one prime ideal in $\widehat{R}$, there exists a prime ideal $\mathbf{p} \subseteq \widehat{R}$ such that $\mathbf{q}=\mathbf{p} \cap D$. We obtain inclusions

$$
R /(\mathbf{p} \cap R) \subseteq D / \mathbf{q} \subseteq \widehat{R} / \mathbf{p}
$$

By assumptions the ring $R /(\mathbf{p} \cap R)$ is complete, and therefore the above inclusions are equalities and $D / \mathbf{q}=\widehat{R} / \mathbf{p}$. Thus, $D / \mathbf{q}=R /(\mathbf{q} \cap R)$, so $D$ is Noetherian by (2.10), and we conclude that $D$ is a normal local Noetherian domain. Moreover, if $a \in \mathbf{m}-\left(\mathbf{p}_{1} \cap \cdots \cap \mathbf{p}_{n}\right)$, then $R / a R$ is complete and $a \widehat{R} \cap D=a D$. Therefore, $R / a R=D / a D-\widehat{R} / a \widehat{R}$. It follows that $\mathbf{m} D$ is the maximal ideal of $D$, and hence that $\widehat{D}=\widehat{R}$.

Corollary 2.12. Suppose $(R, \mathbf{m})$ is an excellent normal local Henselian domain with $\alpha(R)=0$. If $R$ is complete with respect to the a-adic topology for some nonzero ideal a $\subset R$, then $R$ satisfies condition (NIR).

Proof. Using Corollary 1.13, and a similar argument as in the proof of Corollary 1.6, we see that $R$ satisfies condition (CHI), so Theorem 2.11 applies.

Example 2.13. Let $R_{s}[[w]]$ be the excellent regular local ring of Example 1.7. Its Henselization $R_{s}[[w]]^{h}$ satisfies the assumptions of Corollary 2.12 and thus satisfies condition (NIR).

\section{ACKNOWLEDGMENT}

This paper is an outgrowth of a general project that we have been pursuing in collaboration with Judy Sally. We would like to acknowledge her help.

\section{REFERENCES}

[AN] M. André, Cinq exposés sur la désingularisation (in preparation).

[A] M. Artin, Algebraic approximation of structures over complete local rings, Inst. Hautes Études Sci. Publ. Math. 36 (1969), 25-58.

[BKKN] R. Berger, R. Kiehl, E. Kunz, and H. J. Nastold, Differentialrechnung in der analytischen Geometrie, Lecture Notes in Math., vol. 38, Springer-Verlag, Berlin and New York, 1967.

[C] I. S. Cohen, Lengths of prime ideal chains, Amer. J. Math. 76 (1954), 654-668.

[G] A. Grothendieck, Élements de Géometrie algebrique IV $_{2}$, Inst. Hautes Études Sci. Publ. Math. 24 (1965).

[HR] W. Heinzer and C. Rotthaus, On a certain class of algebraically independent elements (in preparation). 
[HRS] W. Heinzer, C. Rotthaus, and J. Sally, Formal fibers and birational extensions (in preparation).

[M1] H. Matsumura, Commutative ring theory, Cambridge Univ. Press, Cambridge, 1986.

[M2] _ On the dimension of formal fibers in a local ring, Algebraic Geometry and Commutative Algebra in Honor of Masayoshi Nagata, Kinokuniya, Tokyo, 1988.

[M3] _ Commutative algebra, second ed., Benjamin/Cummings, Reading, MA, 1980.

[N] M. Nagata, Local rings, Wiley, New York, 1962.

[Ni] Jun-Ichi Nishimura, Note on Krull domains, J. Math. Kyoto Univ. 15 (1975), 397-400.

[P1] D. Popescu, General Néron desingularization, Nagoya Math. J. 100 (1985), 97-126.

[P2] General Néron desingularization and approximation, Nagoya Math. J. 104 (1986), $85-115$.

[R1] C. Rotthaus, On the approximation property of excellent rings, Invent. Math. 88 (1987), 39-63.

[R2] - Rings with approximation property, Math. Ann. 287 (1990), 455-466.

[R3] _ On rings with low dimensional formal fibres, J. Pure Appl. Algebra 71 (1991), 287-296.

[V] P. Valabrega, On two-dimensional regular local rings and a lifting problem, Ann. Scuola Norm. Sup. Pisa Cl. Sci. (4) 27 (1973), 1-21.

Department of Mathematics, Purdue University, West Lafayette, Indiana 47097

E-mail address: heinzer@math.purdue.edu

Department of Mathematics, Michigan State University, East Lansing, Michigan 48824

E-mail address: 21144 cor@msu.edu 